\title{
Surface plasmon resonance monitoring of temperature via phase measurement
}

\author{
H.-P. Chiang ${ }^{\text {a,b,* }}$, H.-T. Yeh ${ }^{\text {b }}$, C.-M. Chen ${ }^{\text {b }}$, J.-C. Wu ${ }^{\text {b }}$, S.-Y. Su ${ }^{\text {b }}$, R. Chang ${ }^{\text {, }}$, \\ Y.-J. Wu ${ }^{\text {b }}$, D.P. Tsai ${ }^{c}$, S.U. Jen ${ }^{\text {a,b }}$, P.T. Leung ${ }^{\text {a,d }}$ \\ ${ }^{a}$ Institute of Physics, Academia Sinica, Taipei, Taiwan, ROC \\ ${ }^{\mathrm{b}}$ Institute of Optoelectronic Sciences, National Taiwan Ocean University, 2 Pei Ning Rd., Keelung 202, Taiwan, ROC \\ ${ }^{\mathrm{c}}$ Department of Physics, National Taiwan University, Taipei, Taiwan, ROC \\ ${ }^{\mathrm{d}}$ Department of Physics, Portland State University, P.O. Box 751, Portland, OR 97207-0751, USA
}

Received 27 April 2004; received in revised form 17 July 2004; accepted 20 July 2004

\begin{abstract}
The application of surface plasmon resonance to the monitoring of the temperature of a metal film and its environment is well-established. A new feature in our present experimental work is to carry out this monitoring via the measurement of the phase difference between a s and a p-polarized wave at different wavelengths, $632.8 \mathrm{~nm}$ and $1.15 \mu \mathrm{m}$, based on a technique established previously in the literature. By monitoring the change of this phase as a function of the film temperature, it is found that this approach leads to very sensitive measurements of temperature, in comparison with previous approaches in which reflectance was measured instead. Sensitivity is $0.027 \mathrm{~K}$ at incident wavelength of $632.8 \mathrm{~nm}$ and $0.1 \mathrm{~K}$ at incident wavelength of $1.15 \mu \mathrm{m}$. A simple model based on the temperature dependence of the optical constants of the metal is applied to simulate our measurements, and it is found that the general qualitative behavior and trend of the experimental results can be reasonably accounted for using such a model.
\end{abstract}

(C) 2004 Elsevier B.V. All rights reserved.

Keywords: Surface plasmon resonance; Temperature effects; Phase measurement

\section{Introduction}

Surface plasmon (SP) refers to the collective oscillation of the free electrons at a metal-

\footnotetext{
* Corresponding author. Fax: +88 6224634360.

E-mail address: hpchiang@mail.ntou.edu.tw (H.-P. Chiang).
}

dielectric interface. Ever since the first introduction of the attenuated total reflection (ATR) method for the optical excitation of these SP's [1], it has been recognized that such excitation can be utilized to monitor with high sensitivity a large variety of physical/chemical processes at interfaces. In the last two decades, various sensing 
techniques based on this mechanism have been developed including, for example, biosensing [2], film-thickness sensing [3], laser-ablation monitoring [4], and temperature sensing [4-6]. These surface plasmon resonance (SPR) sensors all share with some distinct features such as non-invasive nature and capability of real-time monitoring.

The first application of SPR to temperature monitoring was due to Heminghaus and Leiderer [5], in an experiment in which the silver film in an ATR device was heated by a laser pulse. The transient rise in temperature of the metal was monitored by measuring the change of the SPR reflectance (intensity), and an optimal sensitivity of about $2 \mathrm{~K}$ was reported. Other applications of this technique to temperature sensing utilized an adlayer on the metal film in which the optical properties of this adlayer (e.g., a-Si:H) has a much stronger variation with temperature changes compared to that for the metal [6,7]. This approach leads to higher sensitivity in principle, but may more likely be limited to the sensing environment and to lower temperature measurements. Hence in situations under which the monitoring of temperature changes has to depend on the change in optical properties of the metal (e.g., for gaseous systems at high temperatures), an improvement on the approach developed by Heminghaus and Leiderer [5] is desirable.

It is the purpose of our present work to apply the "phase-monitoring technique" established in the literature $[8,9]$, to show that the sensitivity in the direct SPR monitoring of the metal film temperature can be highly improved in this approach. While this phase detection technique has been applied before in the measurement of small changes in refractive index [8] and wavelength shift [9], the capability of this technique has yet to be demonstrated for temperature sensing since all the previous experiments were done via reflectance measurements $[5,6]$. In our recent theoretical work on the sensitivity of temperature-dependent sensitivity of optical sensor based on SPR [10], we have observed that the wavelength of incident light will affect the detection sensitivity. Similar experiment has been carried out by Eum et al. [11]. They have detected the concentration of $\mathrm{K}^{+}$-ion at different incident wavelengths by observing the reflectance dip at resonance angle, and found that higher sensitivity can indeed be achieved with longer wavelengths. Therefore, we would like to investigate if this condition is still valid in phase-monitoring technique by performing the experiment at different wavelengths of $632.8 \mathrm{~nm}$ and $1.15 \mu \mathrm{m}$, respectively. We shall also apply a simple theoretical model to simulate the changes in our SPR signals, and shall see that most qualitative features and trends can be reasonably reproduced in this model. This is possible since the theoretical understanding of the temperature dependence of optical properties for metals is far better than that for other materials, such as those used in approaches with an additional adlayer [6].

\section{Experimental}

The experimental setup, similar to the detection system of [9], is shown in Fig. 1. Unlike the experimental system in [9] which only uses one fixed wavelength light source, two $\mathrm{He}-\mathrm{Ne}$ lasers of wavelengths $632.8 \mathrm{~nm}$ and $1.15 \mu \mathrm{m}$, respectively, have been employed in our system to study the wavelength dependence in phase measurement. A linearly polarized light with angular frequency $\omega_{0}$ from a $\mathrm{He}-\mathrm{Ne}$ laser is introduced through a polarizer. The light is then directed into an electro-optic modulator (ConOptics) with fast axis in the horizontal direction, and then reflected from a quartz prism $\left(n_{\mathrm{p}}=1.452\right)$ coated with a thin metal film which is in contact with a temperature controller. The metal film is coated by using an electron beam evaporator and annealed to optimized condition. The laser light then passes through an analyzer $\mathrm{AN}_{\mathrm{t}}$. Both the transmission axis of the polarizer and analyzer are at $45^{\circ}$ relative to the horizontal direction. The Jones vector of the light after passing through polarizer can be described by

$P=\frac{1}{\sqrt{2}}\left[\begin{array}{l}1 \\ 1\end{array}\right] \mathrm{e}^{\mathrm{i} \omega_{0} t}$.

The Jones matrix of the analyzer is

$\mathrm{AN}_{\mathrm{t}}=\frac{1}{2}\left[\begin{array}{ll}1 & 1 \\ 1 & 1\end{array}\right]$ 


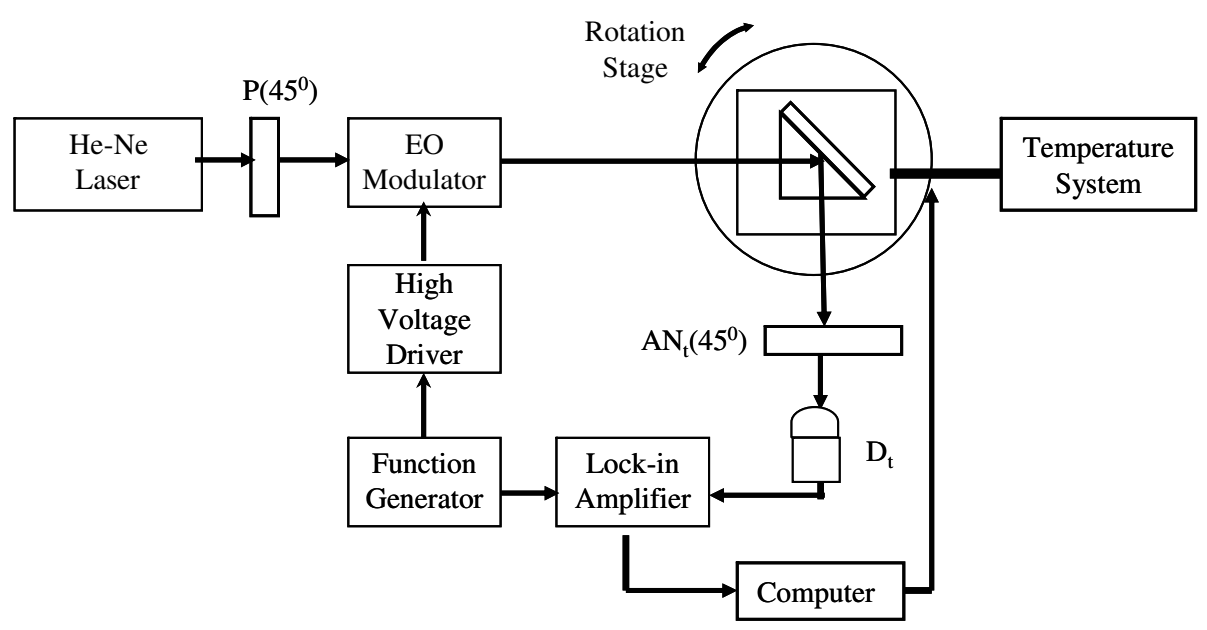

Fig. 1. Experimental setup of phase detection system. P: polarizer; $\mathrm{AN}_{\mathrm{t}}$ : analyzer; $\mathrm{D}_{\mathrm{t}}$ : photodetector.

and that of the metal-film coated prism is

$\mathrm{SPR}=\left[\begin{array}{cc}\left|r_{\mathrm{p}}\right| \mathrm{e}^{\mathrm{i} \phi_{\mathrm{p}}} & 0 \\ 0 & \left|r_{\mathrm{s}}\right| \mathrm{e}^{\mathrm{i} \phi_{\mathrm{s}}}\end{array}\right]$,

where $r_{\mathrm{p}}, r_{\mathrm{s}}, \phi_{\mathrm{p}}, \phi_{\mathrm{s}}$ are the field reflectivity and phases of p-wave and s-wave, respectively. The light is then detected by a photodetector and the converted electric signal from the photodetector is phase-locked by a lock-in amplifier. A sawtooth signal with angular frequency $\omega$ and half-wave voltage $V_{\lambda / 2}$ is applied to the electrooptic modulator. The Jones matrix of the EO modulator is

$\mathrm{EO}=\left[\begin{array}{cc}\mathrm{e}^{\frac{\mathrm{i} \omega t}{2}} & 0 \\ 0 & \mathrm{e}^{-\frac{\mathrm{i} \omega t}{2}}\end{array}\right]$.

Unlike the system in [9], we directly use the sawtooth voltage instead of a photodetected signal from a beam-splitted light for the phase-locking reference. From Jones calculus, the electric field arrived at the photodetector can be described by

$$
\begin{aligned}
E_{\mathrm{t}} & =\mathrm{AN}_{\mathrm{t}} \cdot \mathrm{SPR} \cdot \mathrm{EO} \cdot P \\
& =\frac{1}{2 \sqrt{2}} \cdot\left[\left|r_{\mathrm{p}}\right| \mathrm{e}^{\mathrm{i}\left(\frac{\omega t}{2}+\phi_{\mathrm{p}}\right)}+\left|r_{\mathrm{s}}\right| \mathrm{e}^{\mathrm{i}\left(\frac{(-\omega t}{2}+\phi_{\mathrm{s}}\right)}\right] \cdot\left[\begin{array}{l}
1 \\
1
\end{array}\right] \mathrm{e}^{\mathrm{i} \omega_{0} t}
\end{aligned}
$$

and therefore the corresponding detected intensity is

$$
I_{\mathrm{t}}=\frac{1}{4}\left[\frac{\left|r_{\mathrm{p}}\right|^{2}+\left|r_{\mathrm{s}}\right|^{2}}{2}+\left|r_{\mathrm{p}}\right|\left|r_{\mathrm{s}}\right| \cos \left(\omega t+\phi_{\mathrm{p}}-\phi_{\mathrm{s}}\right)\right] .
$$

Since only p-wave can excite the SPR at a metaldielectric interface $\left|r_{\mathrm{s}}\right|=1$ and $\phi_{\mathrm{s}}$ is almost a constant in Eqs. (1) and (2). We can therefore calculate $\left(\phi_{\mathrm{p}}-\phi_{\mathrm{s}}\right)$ and $r_{\mathrm{p}}$ from Eq. (2).

\section{Results and discussion}

Fig. 2 shows several of our measurements of the angular spectrum of SPR reflectance for a $50 \mathrm{~nm}$ $\mathrm{Ag}$ film at the incident wavelength of $632.8 \mathrm{~nm}$ over the temperature range from 300 to $360 \mathrm{~K}$. Shown also are the theoretical results computed using a previously established model in which the dielectric function of the metal is described by a temperature-dependent Drude model [10]. Note that although the Drude model for free electrons is known to be limited in the account of SPR for silver, it is adopted here since the temperature dependence of the parameters in this model is well-established through various theoretical models (see below). Thus a comparison with experimental results is possible without any fitting parameters. Briefly, we start with the following dielectric function: 

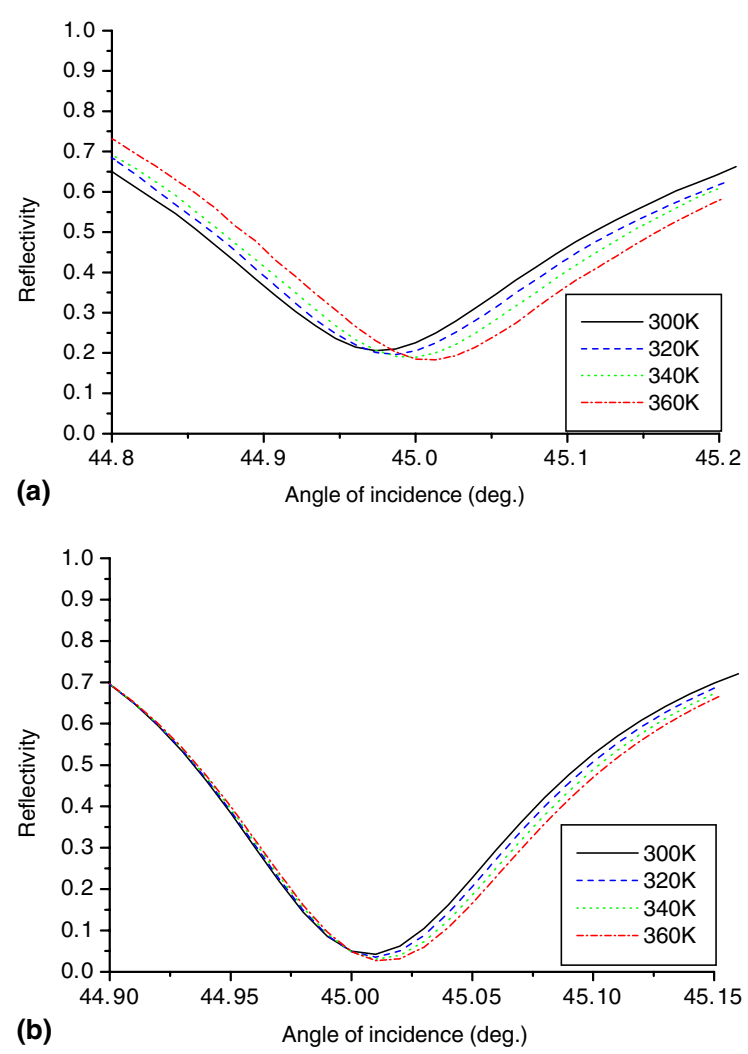

Fig. 2. Reflectance of the temperature-dependent SPR experiment from (a) experiment (b) theory. The thickness of Ag film is $50 \mathrm{~nm}$. The laser wavelength is $632.8 \mathrm{~nm}$ in both cases.

$\varepsilon=1-\frac{\omega_{\mathrm{p}}^{2}}{\omega\left(\omega+\mathrm{i} \omega_{\mathrm{c}}\right)}$,

where $\omega_{\mathrm{c}}$ is the collision frequency and $\omega_{\mathrm{p}}$ the plasma frequency given by

$\omega_{\mathrm{p}}=\sqrt{\frac{4 \pi N e^{2}}{m^{*}}}$

with $N$ and $m^{*}$ the density and effective mass of the electrons, respectively. The collision frequency will have contributions from both phonon-electron and electron-electron scattering

$\omega_{\mathrm{c}}=\omega_{\mathrm{cp}}+\omega_{\mathrm{ce}}$.

As reported previously [10], for a resonance phenomenon like SPR, one must account for the temperature $(T)$ variation of $\omega_{\mathrm{p}}$ besides that for $\omega_{\mathrm{c}}$. As before, $\omega_{\mathrm{p}}$ will depend on $T$ via volumetric effects as follows [10]: $\omega_{\mathrm{p}}=\omega_{\mathrm{p} 0}\left[1+\gamma\left(T-T_{0}\right)\right]^{-1 / 2}$,

where $\gamma$ is the expansion coefficient of the metal, and $T_{0}$ is a reference temperature taken to be the room temperature. $\omega_{\mathrm{c}}$ can then be modeled using the phonon-electron scattering model [12] and the electron-electron scattering model [13], respectively. We thus obtain

$\omega_{\mathrm{cp}}(T)=\omega_{0}\left[\frac{2}{5}+4\left(\frac{T}{\theta}\right)^{5} \int_{0}^{\theta / T} \frac{z^{4} \mathrm{~d} z}{\mathrm{e}^{z}-1}\right]$,

where $\theta$ is the Debye temperature and $\omega_{0}$ is a constant to be determined from the static limit of the above expression together with the knowledge of the d.c. conductivity [10]. In addition, we have

$\omega_{\mathrm{ce}}(T)=\frac{1}{12} \pi^{3} \frac{\Gamma \Delta}{\hbar E_{\mathrm{F}}}\left[\left(k_{\mathrm{B}} T\right)^{2}+(\hbar \omega / 2 \pi)^{2}\right]$,
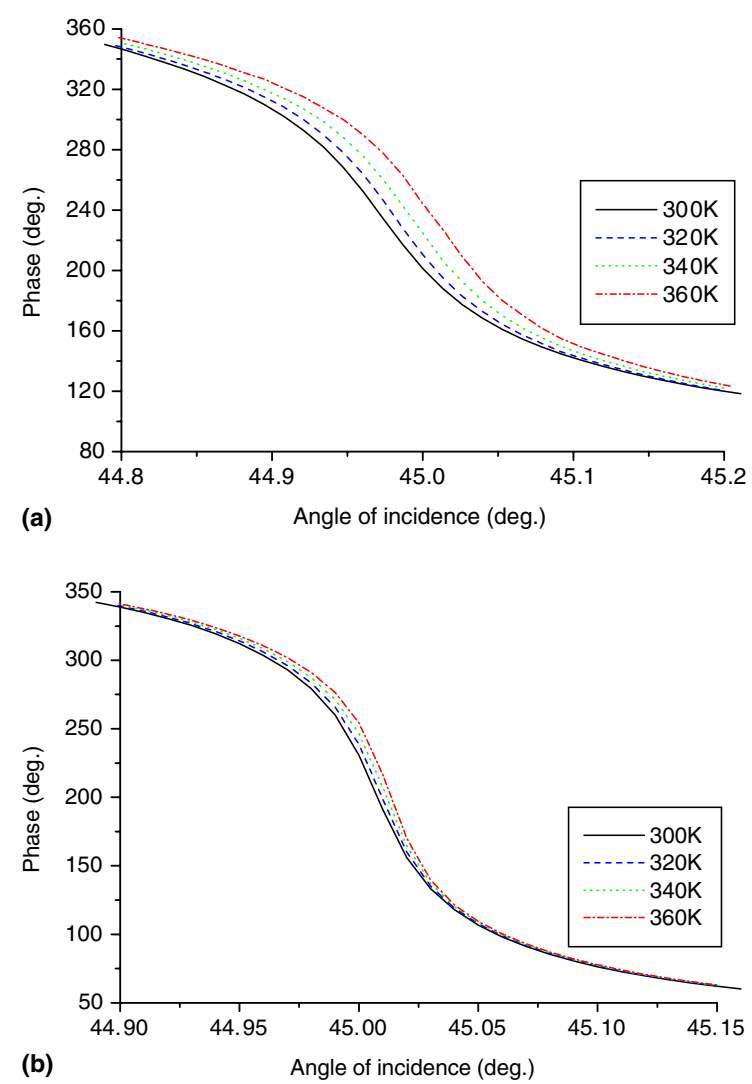

Fig. 3. Phase of the temperature-dependent SPR experiment from (a) experiment (b) theory. The thickness of Ag film is 50 $\mathrm{nm}$. The laser wavelength is $632.8 \mathrm{~nm}$ in both cases. 
where $\Gamma$ and $\Delta$ are defined in [10]. Thus Eqs. (3)(8) together with the well-known Fresnel equations provide a complete model for the simulation of the SPR sensor response to the variation of the temperature of the environment. The reflectance and phase are then calculated by using the Fresnel formulas. It is seen that the measured changes are in general greater than those obtained from the model, though the qualitative trends are agreeable between the two. Fig. 3 shows the results for phase versus angle of incidence for the same experiment as in Fig. 2, and once again only qualitative agreement is found between experiment and theory. Figs. 4 and 5 show results similar to those in Figs. 2 and 3 , but for laser wavelength at $1.15 \mu \mathrm{m}$. We have also performed similar studies using a $\mathrm{Au}$ film, and observed the results from $\mathrm{Ag}$ and $\mathrm{Au}$ are comparable qualitatively. $\mathrm{Ag}$ has a greater
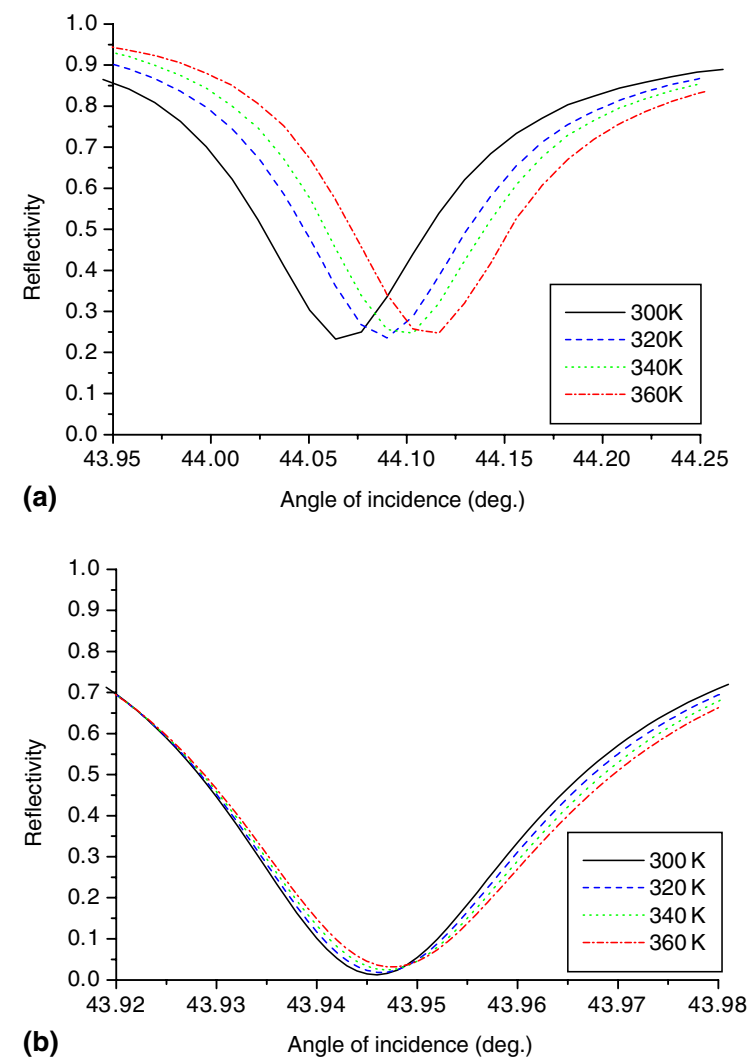

Fig. 4. Reflectance of the temperature-dependent SPR experiment from (a) experiment (b) theory. The thickness of Ag film is $50 \mathrm{~nm}$. The laser wavelength is $1.15 \mu \mathrm{m}$ in both cases. sensitivity to temperature change in these SPR experiments.

The behaviors of phase change near the resonance angle are quite different at different incident wavelengths, as shown in Fig. 3 for $632.8 \mathrm{~nm}$ and Fig. 5 for $1.15 \mu \mathrm{m}$, respectively. In Fig. 3, the phases near resonance angle change from higher to lower values, while the reverse takes place in Fig. 5. Similar results have been observed by the simulation work of Kabashin et al. [14]. They computed the reflectance and phase changes at different thickness of metal films in SPR sensors and found that the trends of phases changed abruptly when the condition for optimized film thickness leading to minimum reflectance dip is achieved. We here study further this phenomenon theoretically by fixing the film thickness at $50 \mathrm{~nm}$ and varying the wavelength of the incident light. Fig. 6(a)
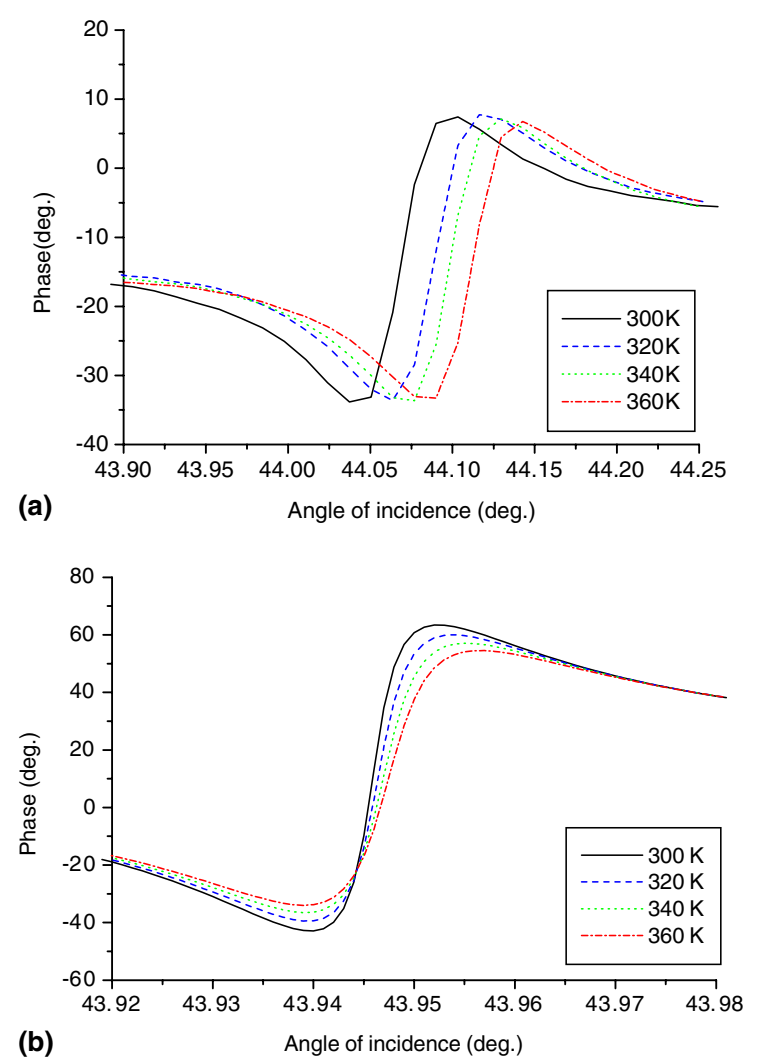

Fig. 5. Phase of the temperature-dependent SPR experiment from (a) experiment (b) theory. The thickness of Ag film is 50 $\mathrm{nm}$. The laser wavelength is $1.15 \mu \mathrm{m}$ in both cases. 
illustrates the reflection coefficient as a function of incident angle for various wavelengths at room temperature. The position of dip shifts towards small angle and the width of the reflectance curve shrinks as the wavelength increases as seen in Fig. 6(a). At a critical wavelength $\lambda_{\mathrm{c}} \sim 974 \mathrm{~nm}$, the reflection at the dip vanishes and the energy of incident light is completely coupled to the surface plasmon which then dissipates into the metal film. The phase of the reflection amplitude corresponding to each wavelength is displayed in Fig. 6(b). In case of short wavelength $(632.8 \mathrm{~nm})$, we observe a large phase change $\left(\sim 340^{\circ}\right)$ in the neighborhood of the angle where the dip occurs. This change becomes steeper as the wavelength approaches $\lambda_{\mathrm{c}}$. The most remarkable feature is that the angle dependence of phase changes abruptly as the wavelength crosses the critical value $\lambda_{\mathrm{c}}$.
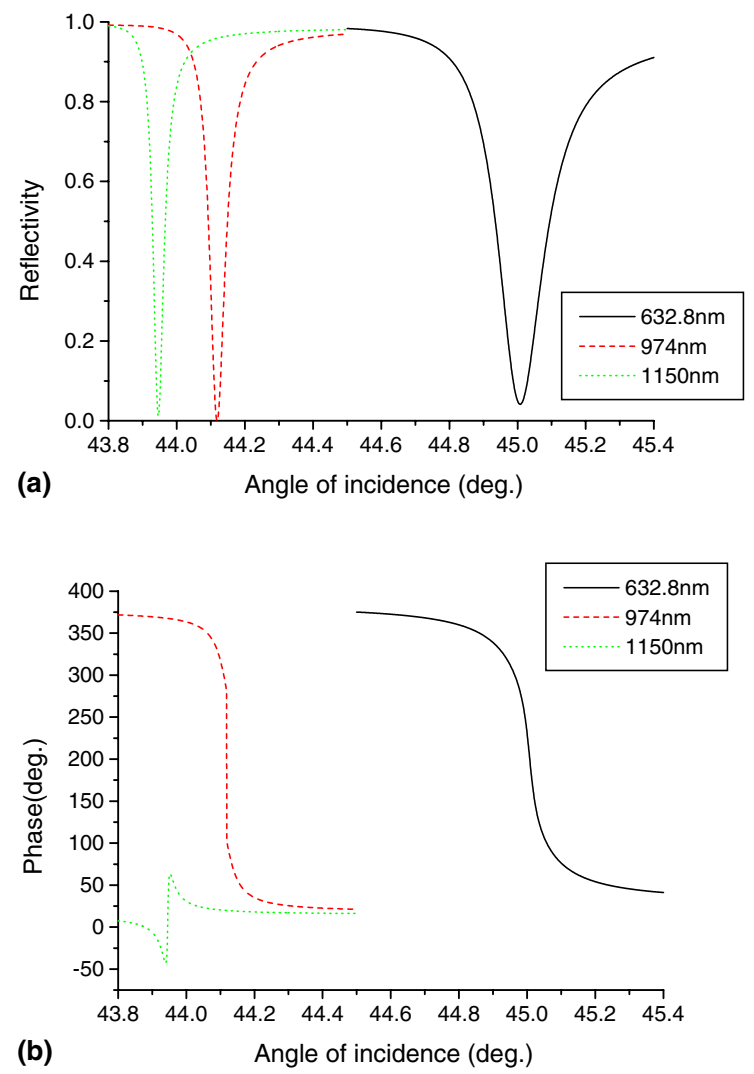

Fig. 6. (a) Reflection coefficient (b) phase as a function of incident angle for various wavelengths at room temperature.
In order to gain more insight into this phenomenon, we have plotted the reflection amplitude $\left(r_{\mathrm{p}}\right)$ on complex plane as the angle of incidence is varied.
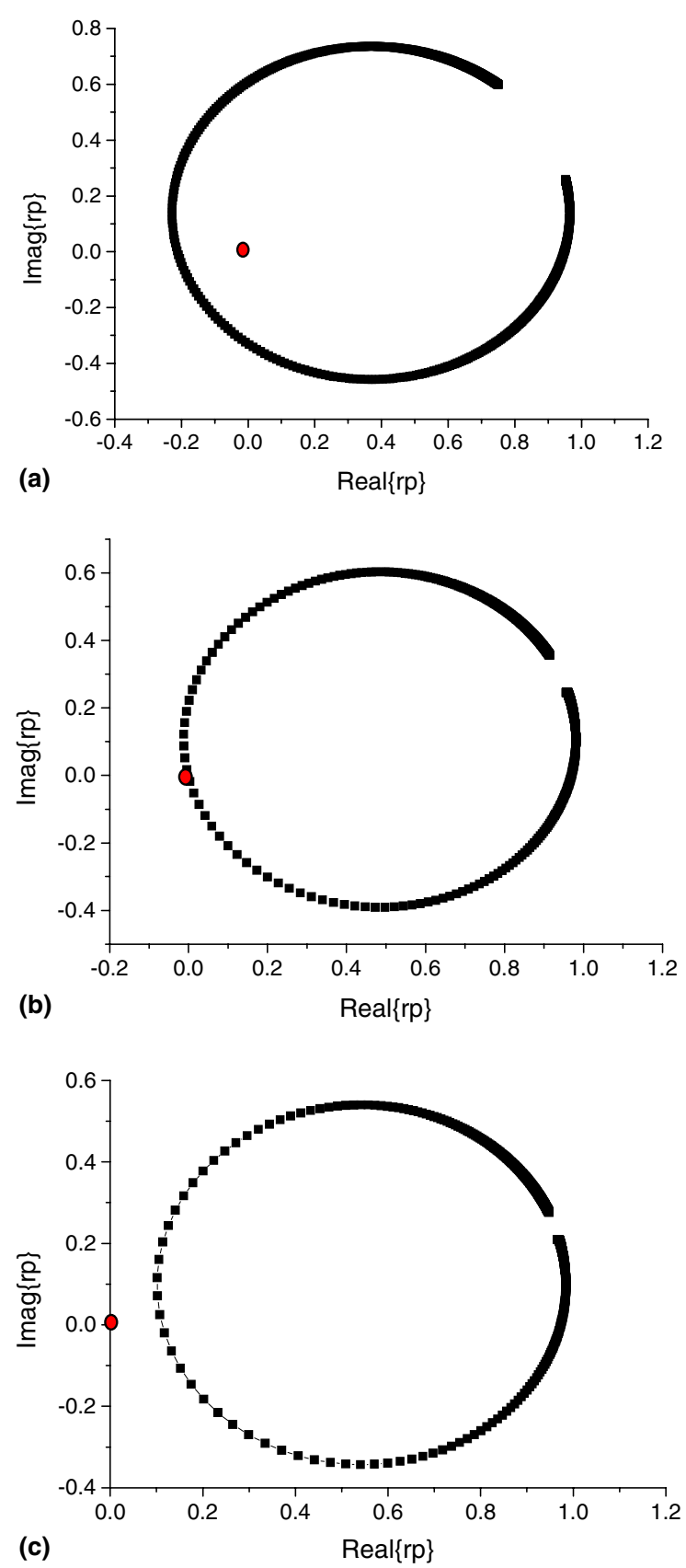

Fig. 7. The plot of reflection amplitude on complex plane for various wavelengths (a) $632.8 \mathrm{~nm}$, (b) $974 \mathrm{~nm}$, and (c) $1.15 \mu \mathrm{m}$ at room temperature as the angle is varied. 

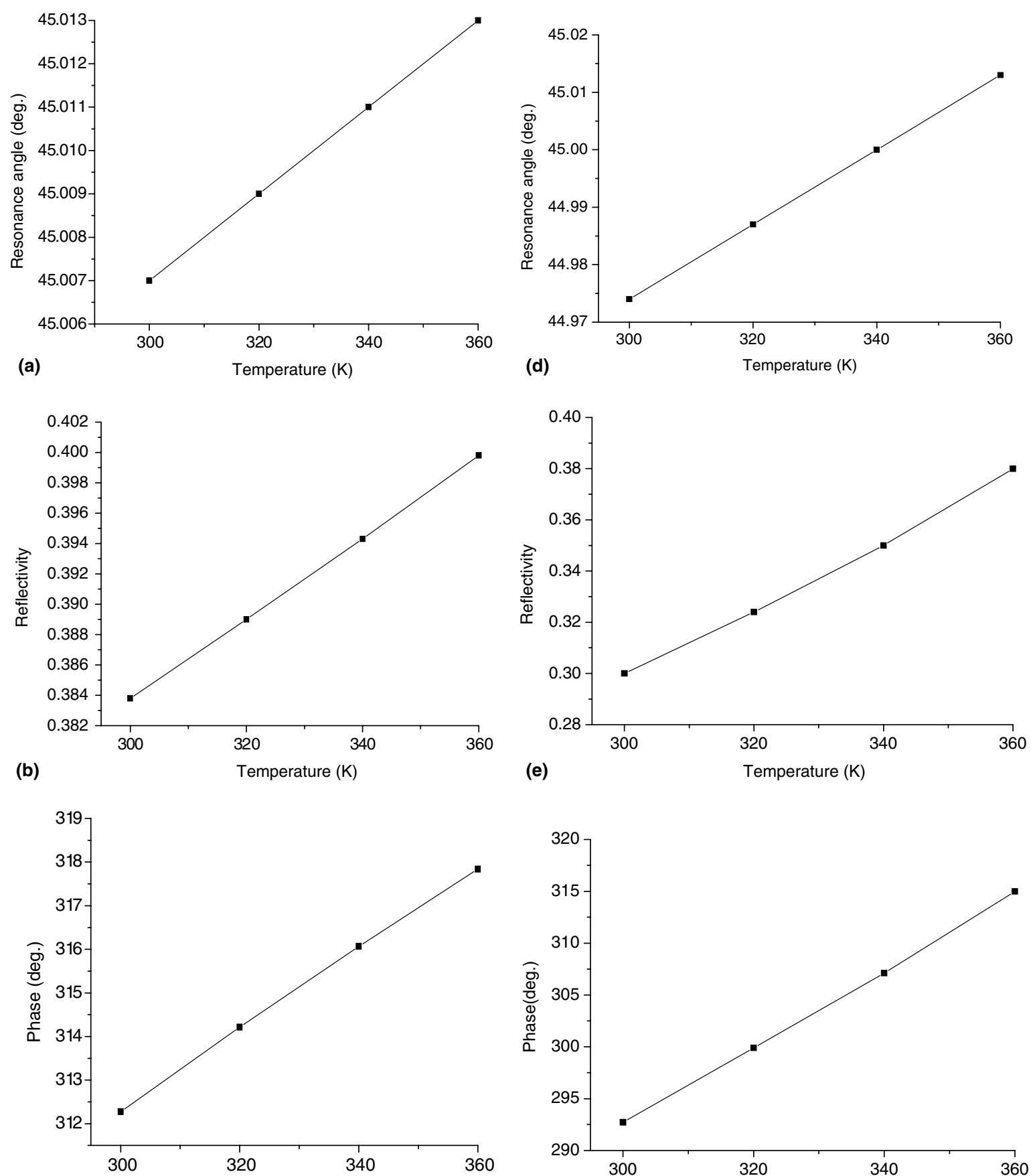

(c)

Temperature $(\mathrm{K})$

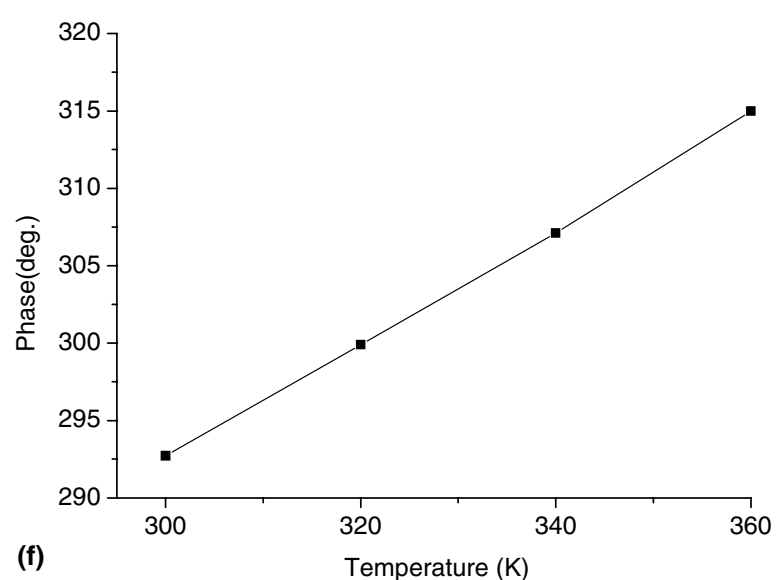

Fig. 8. (a) $R$ vs. $T$, (b) resonance angle vs. $T$, (c) phase vs. $T$, all for $\mathrm{Ag} 50 \mathrm{~nm}$ at wavelength of $632.8 \mathrm{~nm}$. (a)-(c) are from theoretical results and the corresponding experimental graphs are shown in (d)-(f). 

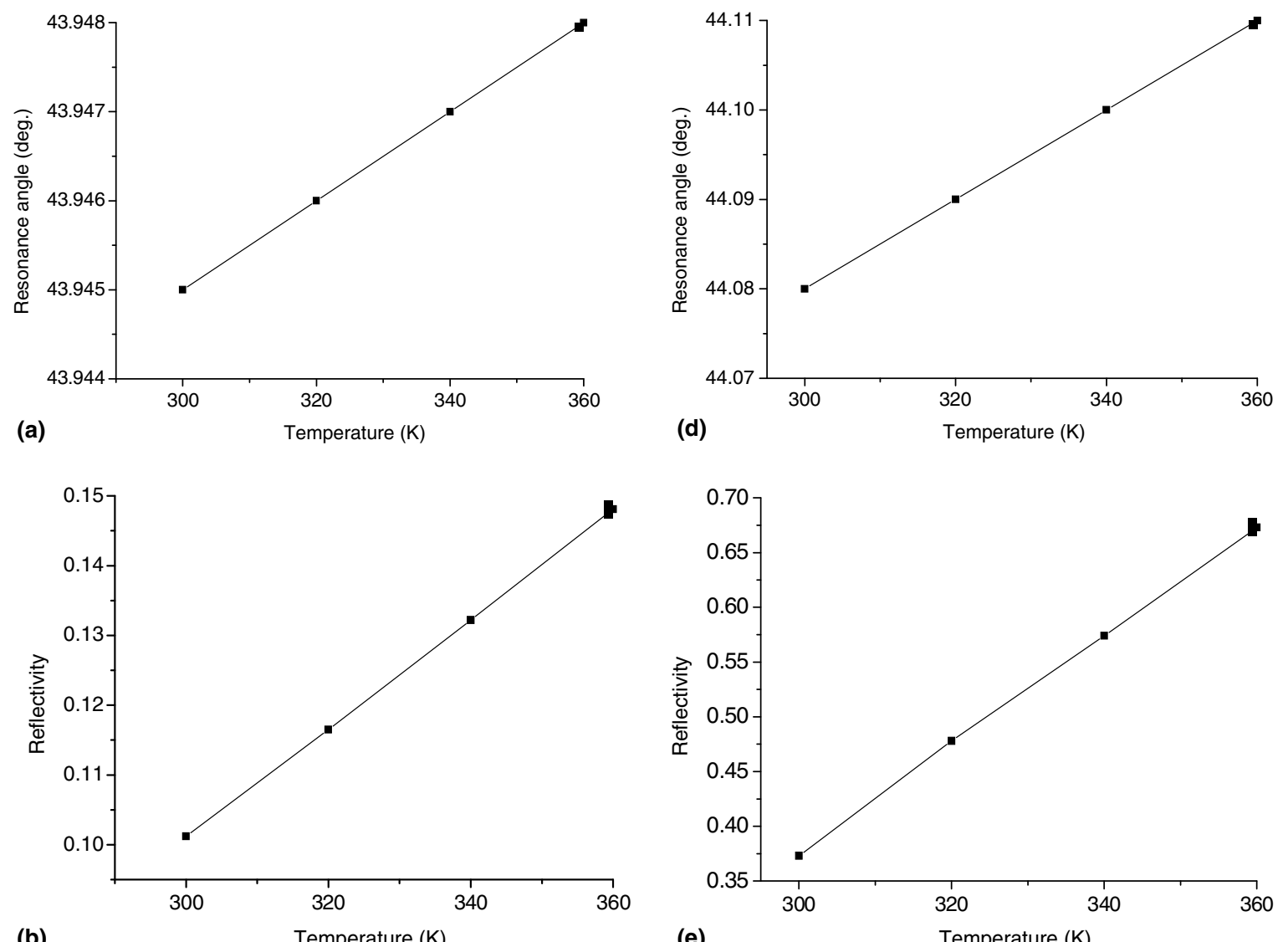

(b)

(e)

Temperature (K)
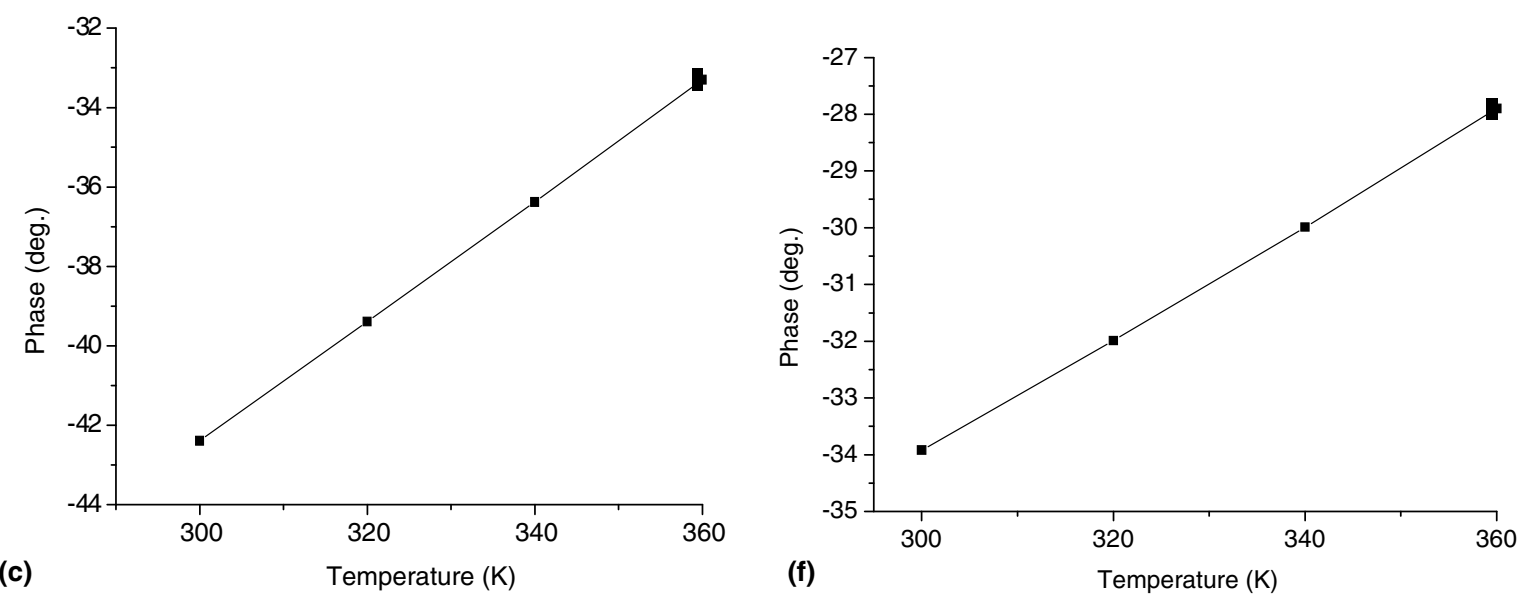

Fig. 9. Similar to Fig. 8, but at wavelength of $1.15 \mu \mathrm{m}$.

The results are shown in Fig. 7. In all cases, the trajectories are loops almost closed. For the short wavelength $(632.8 \mathrm{~nm})$, the loop encloses the origin $(0,0)$ and thus acquires a large phase change over the angle considered. Near the critical wavelength reflection coefficient is almost zero at the dip as shown in Fig. 
6(a) and thus the loop almost passes through the origin, making a phase jump $\sim 180^{\circ}$ as far as the chosen precision of angle is concerned. The jump can be either positive or negative depending sensitively on whether the origin is enclosed or not. One can easily see that as long as the wavelength is larger than $\lambda_{\mathrm{c}}$, e.g., $1.15 \mu \mathrm{m}$, the origin falls outside the loop and leads to the "reverse pattern" as shown in Fig. 6(b). A phenomenon closely resembling to this result was observed recently in a theoretical study of the transmission experiment on quantum dot [15].

The physical origin of this "cross over behavior" in the phase is complicated and depends crucially on two damping rates, namely, the internal damping $\left(\Gamma_{\mathrm{i}}\right)$ and the radiative damping $\left(\Gamma_{\mathrm{rad}}\right)$ as defined in [1]. It can be shown that the reflectivity minimum will be identically zero only if $\Gamma_{\mathrm{i}}=\Gamma_{\mathrm{rad}}$ [1]. The "cross over behavior" then takes place from the case with $\Gamma_{\mathrm{i}}<\Gamma_{\text {rad }}$ (for short wavelength incidence) to the case with $\Gamma_{\mathrm{i}}>\Gamma_{\text {rad }}$ (for long wavelength incidence). Since the wavelength dependence of $\Gamma_{\mathrm{i}}$ and $\Gamma_{\text {rad }}$ is very complicated in general [1], the above account can only be quantitatively illustrated via some detailed model calculations by adopting certain simple description of the dielectric function of the metal film.

In order to compare the sensitivity in temperature measurement among various approaches, we shall follow Nelson et al. [8] to consider the following quantity:

$\sigma_{\mathrm{T}}=\frac{\Delta T}{\Delta p} \sigma_{\mathrm{p}}$,

where $p$ stands for the parameter being monitored during the SPR measurement of the change of temperature, $\sigma_{\mathrm{p}}$ the finest "resolution" available from the equipment used in our experiments, and $\Delta T / \Delta p$ the local slope of the $T$ vs. $p$ curve. From the results shown in Figs. 2-5, we have performed an analysis of the sensitivity in Eq. (9) by referring to (i) angle $(\theta)$ interrogation, (ii) reflectance $(R$, intensity) monitoring, and (iii) phase $(\phi)$ modulation. We note that in (i) it is the value of the resonance angle corresponding to the dip in the reflectance curve which is monitored.

Fig. 8 shows the results of the above three monitoring schemes for the experiment with a $50 \mathrm{~nm}$ $\mathrm{Ag}$ film at $632.8 \mathrm{~nm}$. Both experimental and theoretical results are obtained to follow an almost linear behavior. Hence the local slope in Eq. (9) for this experiment is constant throughout the range of temperature considered in the experiment. Fig. 9 shows the corresponding results for the experiment with laser wavelength at $1.15 \mu \mathrm{m}$. Since the theoretical results for this case still follow closely a linear relationship, we have applied linear regression to obtain the best fit to the experimental data. We have presented in Table 1 a summary of the results for the sensitivities in Eq. (9) computed from the graphs in Figs. 8 and 9. Results computed from both the experimental and theoretical local slopes are shown. Sensitivity is obtained to be $0.027 \mathrm{~K}$ at incident wavelength of $632.8 \mathrm{~nm}$ and $0.1 \mathrm{~K}$ at incident wavelength of $1.15 \mu \mathrm{m}$. From the comparison in Table 1, it is clear that the phase monitoring technique does provide the best sensitivity among the three techniques for SPR temperature measurements. As a final note, we want to remark that in spite of these possible fine temperature resolutions, our results are plotted at a temperature

Table 1

Comparison of the sensitivities among detection methods

\begin{tabular}{|c|c|c|c|}
\hline Modulation technique & Local slope & Instrument resolution $\left(\sigma_{\mathrm{p}}\right)$ & Calculated resolution $\left(\sigma_{\mathrm{T}}\right)$ \\
\hline \multicolumn{4}{|c|}{ (a) Ag $50 \mathrm{~nm}$ and wavelength at $632.8 \mathrm{~nm}$} \\
\hline Angle & $1538 \mathrm{~K} /{ }^{\circ}$ & $0.001^{\circ}$ & $1.538 \mathrm{~K}$ \\
\hline Reflectivity & $688 \mathrm{~K}$ & 0.001 & $0.688 \mathrm{~K}$ \\
\hline Phase & $2.7 \mathrm{~K} /{ }^{\circ}$ & $0.01^{\circ}$ & $0.027 \mathrm{~K}$ \\
\hline \multicolumn{4}{|c|}{ (b) Ag $50 \mathrm{~nm}$ and wavelength at $1.15 \mu \mathrm{m}$} \\
\hline Angle & $1154 \mathrm{~K} /{ }^{\circ}$ & $0.001^{\circ}$ & $1.154 \mathrm{~K}$ \\
\hline Reflectivity & $162 \mathrm{~K}$ & 0.001 & $0.162 \mathrm{~K}$ \\
\hline Phase & $10 \mathrm{~K} /{ }^{\circ}$ & $0.01^{\circ}$ & $0.1 \mathrm{~K}$ \\
\hline
\end{tabular}


interval of $20 \mathrm{~K}$. This is done to exhibit clear visual distinction of various results over a large temperature range.

\section{Conclusion}

We have demonstrated that by monitoring the relative phase change between a $s$ and a $p$ reflected wave in the application of SPR to temperature measurements, great sensitivity can be achieved (down to $0.027 \mathrm{~K}$ ) in spite of the relative small change of optical response with temperature for metal. This is consistent with previous phase monitoring in the SPR measurements of small changes in reflective index [8] and in wavelength shift [9] in which high sensitivity via this approach were again obtained. It has been pointed out that this phase technique gains in sensitivity at the expense of dynamical range in the measurement of the quantity of interest [8]. But since the variation in optical properties of metal with temperature is extremely modest over a reasonable large range of temperature values, this technique may lead to high sensitivity with very little sacrifice in the dynamical range in temperature measurements. We thus advocate that this phase monitoring technique to be implemented in any SPR temperature sensor.

\section{Acknowledgements}

This research is partially supported by the National Science Council of Taiwan under Grant
Nos. NSC92-2112-M-019-006, NSC93-2120-M019-001, the Center for Nanostorage Research at National Taiwan University under Grant No. MOEA\#92-EC-17-A-08-S1-0006 and the PSU Faculty Development Committee.

\section{References}

[1] H. Raether, Surface Plasmons on Smooth and Rough Surfaces and on Gratings, Springer, Berlin, 1988.

[2] See, e.g., J. Homola, Anal. Bioanal. Chem. 377 (2003) 528.

[3] See, e.g., L.S. Jung, C.T. Campbell, T.M. Chinowsky, M.N. Mar, S.S. Yee, Langmuir 14 (1998) 5636.

[4] S. Herminghaus, P. Leiderer, Appl. Phys. Lett. 58 (1991) 352.

[5] S. Herminghaus, P. Leiderer, Appl. Phys. A 51 (1990) 350.

[6] B. Chadwick, M. Gal, Jpn. J. Appl. Phys. 32 (1993) 2716.

[7] See also the small temperature change observed in a water/ ethanol adlayer as reported by K. Matsubara, S. Kawata, S. Minami, Appl. Spectrosc. 42 (1988) 1375.

[8] S.G. Nelson, K.S. Johnson, S.S. Yee, Sensor Actuat. 3536 (1996) 187.

[9] K.H. Chen, C.C. Hsu, D.C. Su, Opt. Commun. 209 (2002) 167.

[10] H.-P. Chiang, Y.C. Wang, P.T. Leung, W.S. Tse, Opt. Commun. 188 (2001) 283;

H.-P. Chiang, Y.-C. Wang, P.T. Leung, Thin Solid Film 425 (2003) 135.

[11] N.-S. Eum, S.H. Lee, D.-R. Lee, D.-K. Kown, J.-K. Shin, J.-H. Kin, S.-W. Kang, Sensor Actuat. B 96 (2003) 446.

[12] T. Holstein, Phys. Rev. 96 (1954) 535; Ann. Phys. (NY) 29 (1964) 410.

[13] W.E. Lawrence, Phys. Rev. B 13 (1976) 5316.

[14] A.V. Kabashin, K.E. Kochergin, A.A. Beloglazov, P.I. Nitikin, Biosensor Bioelectron. 13 (1998) 1263.

[15] T. Taniguchi, M. Buttiker, Phys. Rev. B 60 (1999) 13814. 